\title{
A Review on Comparison of Complete and Incomplete Block Designs
}

\author{
Habtamu Kefale Sewenet \\ College of Agriculture and Natural Resources, Department of Plant Science, Debre Markos University, Debre \\ Markos, Ethiopia
}

\begin{abstract}
The study of strategies for efficient plans for the collection of data, which lead to proper estimates of parameters relevant to the researcher's objective is known as experimental design. Field-based agronomic and genetic research is a decision-based process and many of decisions are required to decide the type of design used to conduct a field experiment, collect, analyze data and interpret the results. Many researchers are locked into particular dimensions that are partly determined by the size or capacity of planting or harvesting equipment, available resources, the size and shape of fields available for research, and colleagues' perceptions, or perhaps even peer pressure. A properly designed experiment for a particular research objective is the basis of all successful experiments. Experimental designs are classified in to complete block and incomplete block designs based on the treatment numbers found in a block. In case of complete block designs, all treatments are found in a block while some treatments found in incomplete block designs. Some of complete block designs are, completely randomized design (CRD), completely randomized block design (RCBD, Latin square (LSD), split plot design (SPD) whereas incomplete block designs are lattice design (LD) and Augmented designs (AD) are most known. Most of the time complete block design is widely used by researchers more than the balanced incomplete block designs because the missing data are computed before analysis. Each research designs had their own level of precision and conditions. Therefore, no single design is best all over designs. Due to this, the present review highlights the basic comparison concepts of complete and incomplete experimental designs and the focus of selection criteria.
\end{abstract}

Keywords: Complete block, Experimental design and Incomplete block

DOI: $10.7176 / \mathrm{JBAH} / 9-9-03$

Publication date:May $31^{\text {st }} 2019$

\section{INTRODUCTION}

Experimentation plays a momentous role in the field of agriculture. A good experiment is the one which involves good planning, accurate data collection, proper data analysis and precise interpretation of the data (Masood et al., 2008). Experimental design is the process of planning a study to meet specified objectives. Planning an experiment properly is very important in order to ensure that the right type of data and a sufficient sample size and power are available to answer the research questions of interest as clearly and efficiently as possible.

Field-based agronomic and genetic research is a decision-based process and many of decisions are required to decide the type of design used and conduct a field experiment, collect and analyze the data, and interpret the results (Casler, 2013). According to his study large number of these decisions have nothing to do with the hypotheses to be tested, but instead relate to the design of the experimental arrangement used to create valid and convenient hypothesis tests. Most of these decisions are made using one or both of two broad criteria. First, many elementary statistical textbooks and a few journal articles offer some general guidelines on broad concepts of field-plot trial design, such as when and how to use blocking designs, various methods of implementing randomization restrictions, and data analysis methodology (Box et al., 2005). Second, equipment dimensions, convenience, and personal preferences drive many decisions, particularly size and shape of experimental units and blocks (Hinkelmann and Kempthorne, 2008).

Many researchers are locked into particular dimensions that are partly determined by the size or capacity of planting or harvesting equipment, the size and shape of fields available for research, and colleagues' perceptions, or perhaps even peer pressure. The study of strategies for efficient plans for the collection of data, which lead to proper estimates of parameters relevant to the researcher's objective, is known as experimental design (Lentner and Bishop, 1993). A properly designed experiment, for a particular research objective, is the basis of all successful experiments. Most of the time complete block design is widely used by researchers more than the balanced incomplete block designs because the missing data are computed before analysis (Kelechi, 2012).

Experimental designs are basically divided into two categories: Complete block designs (blocks are complete) and incomplete block designs-(blocks are incomplete). But there is no one best experimental design for all situations. Each design was developed to control variability under a given experimental condition. The choice of an experimental design depends upon the nature of experimental material to be tested and the variability present. However, if an experimenter is given an opportunity to choose a guiding principle to choose an experimental design: 
a) Which is simpler in terms of layout and analysis, and

b) Which design also adequately control variability?

Complete block types of designs are inefficient for large number of treatments, because of their failure to adequately minimize the effect of soil heterogeneity. Generally, the greater the heterogeneity within blocks, the poorer the precision of treatment effect estimates. It is affordable when the block size is less than Twenty treatments (Stringer et al., 2012). Incomplete block designs are arranged in relatively small blocks that contain fewer treatments than the total number of treatments to be compared. Consequently, there is a gain in precision due to use of small blocks. As far as the layout of the experiment is concerned the incomplete block designs are no more difficult than randomized blocks. Some extra planning is involved in drawing up and randomizing the experimental plan. It is always useful to use incomplete block design when the number of varieties/treatments increases. Because of large number of treatments, the homogeneity among experimental units/plots within a large block cannot be maintained. As a result, estimate of experimental error is inflated and results are low in precision (Masood et al., 2008).

Raza and Masood (2009) listed the most commonly used complete block designs for experiments are: Completely randomized design (CRD), Randomized complete block design (RCBD), Latin square design (LD) and split plot design (SPD). Whereas, incomplete block designs are so diverse depending upon types of experiments. In simple experiments, the common incomplete block designs are:

$\checkmark$ Lattice design: - Balanced lattice design (BLD), Partially balanced lattice design (PBLD), they are so diverse: the two replications, the partially balanced lattice design is referred to as a simple lattice; with three replications, a triple lattice; with four replications, a quadruple lattice; and

$\checkmark$ Augmented designs (group balanced block design) - with two groups, a simple augmented design; with three groups, a triple augmented design; with four groups, a quadruple augmented design; and so on.

However, such flexibility in the choice of the number of replications results in a loss of symmetry in the arrangement of treatments over blocks (i.e., some treatment pairs never appear together in the same incomplete block). Consequently, the treatment pairs that are tested in the same incomplete block are compared with a level of precision that is higher than for those that are not tested in the same incomplete block. Because there is more than one level of precision for comparing treatment means, data analysis becomes more complicated (Gomez and Gomez, 1984).

\section{COMPLETE AND INCOMPLETE BLOCK DESIGN COMPARISON}

The experimental field that the researchers use is a heterogeneous environment, mainly due to soil heterogeneity and slope. Soil heterogeneity is a significant source of variance and affects the correct evaluation of treatments genotypes because of the increased experimental error (Gomez and Gomez, 1984). Complete Block Designs attempt to reduce the effect of soil heterogeneity by increasing variability among blocks and minimizing variability within blocks. In this design treatments must be arranged all together in each group, but the number of treatments usually cannot exceed 20, because heterogeneity increases within the block and consequently experimental error increases too (Stringer et al., 2012). To overcome this problem, a new category of designs has been developed with each group divided into smaller more homogeneous subgroups that do not contain all treatments and these designs are called Incomplete Block designs. Commonly known incomplete block designs are augmented and lattice with their sub divisions. Whereas complete block designs are Completely Randomized Design, Completely Randomized Block Design, Latin Squire Design and Split Plot Design are most known and frequently used based on their efficiency and the type of experiment proposed to be done (Katsileros and Koukouvinos, 2015). Field experiments in agronomy and related disciplines have traditionally been affected by soil heterogeneity and in this case treatment effects are small due to soil variability is high, as this inflates the error term (Van Es et al., 2009). According to the Author, the Agronomy Journal reported 537 research efforts, some papers including more than one experiment (Table 1). Of those, $414(77 \%)$ were reported to be field experiments, $37(7 \%)$ were greenhouse trials, and $22(4 \%)$ laboratory efforts and rest are symposium reports, or others (methodology, notes, survey)

Table 1 types and frequency of experiments

\begin{tabular}{|l|l|l|l|}
\hline Type of research & Frequency in number & Frequency in \% & Rank \\
\hline Field based experiment & 417 & 77.65 & 1 st \\
\hline Green house experiment & 37 & 6.89 & $2^{\text {nd }}$ \\
\hline Laboratory experiment & 22 & 4.09 & $4^{\text {th }}$ \\
\hline Modeling/simulations & 20 & 3.73 & $5^{\text {th }}$ \\
\hline Review symposium & 27 & 5.02 & $3^{\text {rd }}$ \\
\hline Others (surveys) & 17 & 3.16 & Last \\
\hline Total & 537 & $100 \%$ & \\
\hline
\end{tabular}

Adopted from: (Van Es et al., 2009)

Out of the 414 field experiments, the majority (300, 72\%) were implemented as RCB designs. Completely 
Randomized, Randomized Incomplete Block, Split Block and Latin Square designs were rarely used (4, 3, 2, and 1 occurrence, respectively). In addition, 9 experiments involved non-randomized field strips, typically involving on-farm research efforts, and 53 involved other field sampling efforts (surveys, etc.).

Although, all research designs are used to conduct scientific agricultural experiments on field and green house, each designs had their own resource requirement, degree of precision, lay out and randomization, number of replication, ANOVA and mean separation, treatment and block size(number).

\subsection{Resource requirement}

Complete block designs require larger experimental resources than incomplete block designs because of all treatments should replicated and found in all blocks. If the number of treatments to be compared is large, then we need large number of blocks to accommodate all the treatments. This requires more experimental material, experimental unit and so the cost of experimentation becomes high which may be in terms of money, labor, time etc. The completely randomized design and randomized block design may not be suitable in such situations because they will require large number of experimental units to accommodate all the treatments. In such situations when sufficient numbers of homogeneous experimental units are not available to accommodate all the treatments in a block, then incomplete block designs can be used. In incomplete block designs, each block receives only some of the selected treatments and not all the treatments. Sometimes it is possible that the available blocks can accommodate only a limited number of treatments due to several reasons (Katsileros et al., 2015).

The popular design for testing accessions is called a "lattice". When lattices are used for genetic resources evaluation, they will often comprise just two replicates, to maximize the number of accessions that can be evaluated on a given area of land and alpha designs are an extension of lattices to blocks with a different number of plots also used. Another incomplete block design is the "augmented design", a type of design that allows land to be used even more efficiently. Augmented designs have just a single replicate of the test accessions. They are therefore of particular value when there is a shortage of seed for the accessions, or of land (IPGRI, 2001).

For example, if you plan to evaluate more than 30 variety of a given crop species for their yield performance in a limited resource, then you have to decide the requirement of resources either in a complete or incomplete designs. So, it is obvious that complete block designs require more resource than incomplete designs because of an increase the treatments will also increase the experimental unit and its respective resources. Similar result was reported by Katsileros et al. (2015).

Table 2. the size of experimental designs

\begin{tabular}{|l|l|l|l|l|l|}
\hline Designs & Treatments & Blocks & Plots & Rep. & Total exp. unit \\
\hline RCBD & 24 & 3 & 24 & 3 & 72 \\
\hline Alpha & 24 & 4 & 6 & 2 & 48 \\
\hline Augmented & $24+4$ & 4 & 10 & - & 40 \\
\hline
\end{tabular}

Source: Katsileros et al. (2015)

The above table is taken on the research done in order to evaluate the efficiency of Incomplete Block (Alpha and Augmented) designs in comparison with the traditional Randomized Complete Block Design (RCBD) in durum wheat trials. So as the table tells us in case of RCBD it had 72 total experimental units where as in an incomplete design had $\leq 48$ total experimental units with the same treatment number.

\subsection{Layout and Randomization}

In a completely randomized design (CRD), a group of experimental units are available and the experimenter randomly assigns treatments to the experimental units. This means there is no randomization restriction or randomization is complete through the experimental area. The data consist of a group of observations on each treatment. Typically, these groups of observations are subjected to a one-way analysis of variance (Christensen, 2013). Incomplete block designs are arranged in blocks that are smaller than a complete replication, in order to eliminate heterogeneity to a greater extent than is possible with randomized blocks; this reduction in the size of block was achieved by sacrificing all, or part of, the information on certain treatment comparisons.

The complete block should be as homogeneous as possible in terms of soil and other environmental factors. The researcher may not know the direction of the soil gradient on the experimental area and may allocate the treatments in the wrong direction. However, it is clear that with the smaller the block size less chance exists of having heterogeneity of soil within the complete block. When the block size is large, the chances of having heterogeneity soil conditions within the block increase. As the number of treatments increases, the chances of finding a homogeneous complete block dramatically decreases and another design is required. It is recommended that the block (or replicates) be as compact as possible (Lentner and Bishop, 1993). The randomization process for complete block designs especially in (RCB design) is applied separately and independently to each of the blocks (Gomez and Gomez, 1984). 


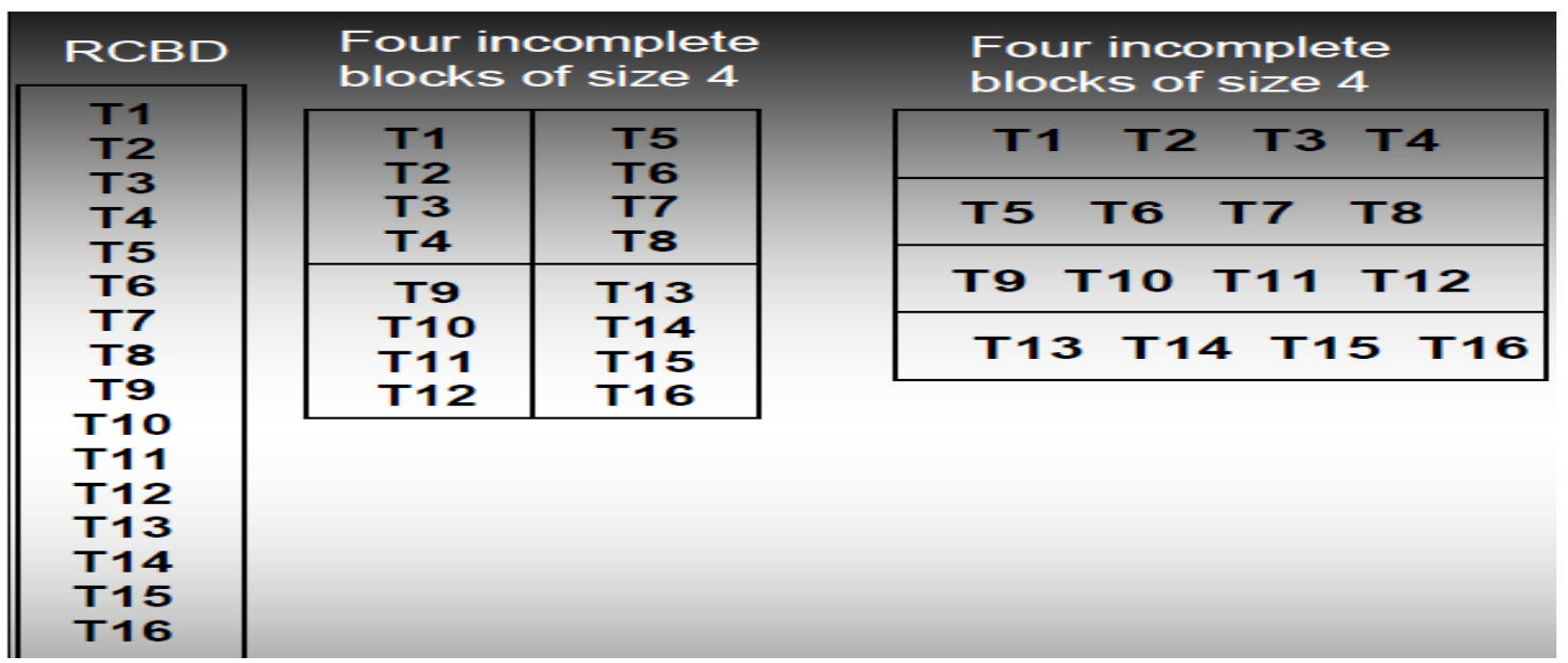

Figure 1 Figure showing the possible lay out in the field of a randomized complete block design (RCBD) and the layout of an incomplete block design of 4 incomplete block size of size 4. Source: (Cochran and Cox, 1957).

Figure (2) shows the possible layout in the field of a randomized complete block design (RCBD) and the layout of an incomplete block design (lattice design) of four incomplete block sizes of size 4. The layout of the RCBD covers a band of the field that accounts for soil gradient on the upper and lower parts of the field. The two possible layouts of the $4 \times 4$ incomplete blocks will control local variability in a much more efficient manner.

\subsection{Treatment number and Block size}

The complete block design types of experiments are inefficient for large number of treatments, because of their failure to adequately minimize the effect of soil heterogeneity (Katsileros. et al., 2015). Generally, the greater the heterogeneity within blocks, the poorer the precision of variety effect estimates. Incomplete block designs are arranged in relatively small blocks that contain fewer varieties than the total number of varieties to be compared. Consequently, there is a gain in precision due to use of small blocks. As far as the layout of the experiment is concerned the incomplete block designs are no more difficult than randomized blocks. Some extra planning is involved in drawing up and randomizing the experimental plan. Randomized Complete Block Design (RCBD) is affordable when the block size is less than twenty varieties/treatments (Masood et al., 2008) and in the other study not more than 16 (Yang et al., 2004). It is always useful to use incomplete block design when the number of varieties/treatments increases. Because of large number of treatments, the homogeneity among experimental units/plots within a large block cannot be maintained. As a result, estimate of experimental error is inflated and results become low in precision (Masood et al., 2008). Although, balanced Lattice and partially Balanced-IBD designs were developed for large numbers of treatments, they require many replications in order to be effective.

One precondition for complete block designs, all treatments must appear in all blocks (For RCBD) and all rows or columns (For LSD) but, sometimes with large number of treatments (say 20 accessions), each requiring relatively large plot sizes, and this condition may not be practicable. In practice, the LS design is applicable only for experiments in which the number of treatments is not less than four and not more than eight. Because of such limitation, the LS design has not been widely used in agricultural experiments despite its great potential for controlling experimental error (Gomez and Gomez 1984). Complete block designs then fail to reduce the effect of heterogeneity, when the number of factors and their levels increase, the number of treatment combinations increase rapidly and it is not possible to participate all these treatment combinations in a single homogeneous block. (Idrees and Khan, 2009).

The designs in which the block phenomenon is followed but the condition of having all the treatments in all blocks is not met are called Incomplete Block designs. In Incomplete Block situations, the use of several small blocks with fewer treatments results in gains in precision but at the expense of a loss of information on comparisons within blocks. Incomplete block designs are now widely used in plant breeding and variety testing around the world. But the analysis of data for incomplete block designs is more complex than complete block design. Thus where computation facilities are limited, incomplete block designs should be considered a last option (Nokoe, 2017).

Complete block designs become less efficient as the number of treatments increases, primarily because block size increases proportionally with the number of treatments, and the homogeneity of experimental plots within a large block is difficult to maintain. That is, the experimental error of a complete block design is generally expected to increase with the number of treatments (Gomez and Gomez, 1984). An alternative set of 
designs for single-factor experiments having a large number of treatments is the incomplete block designs, one of which is the lattice design. As the name implies, each block in an incomplete block design does not contain all treatments and a reasonably small block size can be maintained even if the number of treatments is large. With smaller blocks, the homogeneity of experimental units in the same block is easier to maintain and a higher degree of precision can generally be expected. Augmented designs are appropriate for evaluation stages when hundreds or even thousands of accessions are being studied in the same experiment, using a limited amount of sowing material, perhaps enough for one replicates only (IPGRI, 2001).

As Gomez and Gomez (1984) point of view, there is no concrete rule as to how large the number of treatments should be before the use of an incomplete block design which should be considered, the following guidelines may be helpful:

$\checkmark \quad$ Variability in the Experimental Material. The advantage of an incomplete block design over the complete block design is enhanced by an increased variability in the experimental material. In general, whenever block size in a RCB design is too large to maintain a reasonable level of uniformity among experimental units within the same block, the use of an incomplete block design should be seriously considered. For example, in irrigated rice paddies where the experimental plots are expected to be relatively homogeneous, a RCB design would probably be adequate for a variety trial with as many as, say, 25 varieties. On the other hand, with the same experiment on a dry land field, where the experimental plots are expected to be less homogeneous, a lattice design may be more efficient.

$\checkmark$ Computing Facilities and Services. Data analysis for an incomplete block design is more complex than that for a complete block design. Thus, in situations where adequate computing facilities and services are not easily available, incomplete block designs may have to be considered only as the last measure.

In general, an incomplete block design, with its reduced block size, is expected to give a higher degree of precision than a complete block design. Thus, the use of an incomplete block design should generally be preferred so long as the resources required for its use (e.g., more replications, inflexible number of treatments, and more complex analysis) can be satisfied. The lattice design is the incomplete block design most commonly used in agricultural research. There is sufficient flexibility in the design to make its application simpler than most other incomplete block designs. This section is devoted primarily to two of the most commonly used lattice designs, the balanced lattice and the partially balanced lattice designs. Both require that the number of treatments must be a perfect square (Gomez and Gomez, 1984).

\subsection{Analysis of Variance (ANOVA) and Mean Comparison}

The main technique adopted for the analysis and interpretation of the data collected from an experiment is the analysis of variance technique that essentially consists of partitioning the total variation in an experiment into components ascribable to different sources of variation due to the controlled factors and error. Analysis of variance clearly indicates a difference among the treatment means (Prasad and Gupta, 2014). The analysis of variance (AOV or ANOVA) is defined as the breakdown of variability in to its component parts. It is a statistical technique used to test a hypothesis concerning the means of three or more populations. F-distribution plays a big role in the analysis of variance. The sources of variation could be treatment (experimental error), blocks error etc. and varies with the type of design used.

In an incomplete designs Treatment comparisons are confounded with block effects

- Block differences may affect treatment comparisons

- Block variances could inflate treatment variance.

The analysis of variance is the powerful statistical technique developed for analyzing measurements that depend on several kinds of effects which operate simultaneously, to decide which kind of effects are important and also to estimate these effects. It is a powerful technique, which allows analysis and interpretation of observations from several populations. This versatile statistical tool partitions the total variation in a data set according to the source of variation that is present in an experiment. Historically, the technique of the analysis of variance was developed mainly in connection with problems related to agriculture experimentation. However, today, the technique is widely used in biological, social and industrial experimentation. The ANOVA for two groups is identical to the results obtained with a t-test; it is fair to say that ANOVA is an extension of the $t$ - test to handle more than two independent groups. The theoretical basis for performing the ANOVA test is the partitioning of the available variance of all observations into two sources of variations- variation between the group means and variation within each of the groups. 
Table 3. Designs and their source of variation

\begin{tabular}{|c|c|}
\hline 1. Complete block designs & Source of variation \\
\hline CRD & Treatment, Replication, Error \\
\hline $\begin{array}{l}\text { RCBD } \\
\text { Factorial RCBD }\end{array}$ & 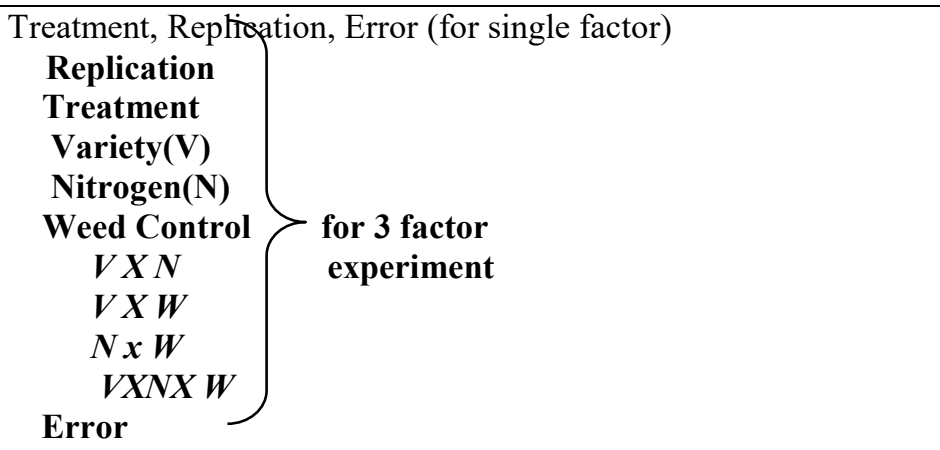 \\
\hline Split Plot & $\begin{array}{l}\text { Replication } \\
\text { Main-plot } \\
\text { factor(A) } \\
\text { Error(a) } \\
\text { Subplot } \\
\text { factor(B) } \\
A X B \\
\text { Error(b) }\end{array}$ \\
\hline $\begin{array}{l}\text { Latin squire } \\
\text { 2. Incomplete block designs }\end{array}$ & Treatment, Column, Row and Error \\
\hline Augmented & $\begin{array}{ll}\text { Block } & (\mathrm{b}-1)=3 \\
\text { Ignoring entries (a) } & \\
\text { Eliminating entries(b) } & \\
\text { Treatment (Entries) } & (\mathrm{C}+\mathrm{P}-1) \\
\text { Adjusted Entries } & \\
\text { Unadjusted entries } & \mathrm{C}-3 \\
\text { Checks } & \mathrm{P}-15 \\
\text { Test Culture } & \\
\text { Test Culture x check } & \\
\end{array}$ \\
\hline Lattice for balanced lattice & Replication, Treatment, Incomplete block, and Experimental error. \\
\hline For partially balanced lattice & Replication, treatments, incomplete block and experimental error \\
\hline
\end{tabular}

Source: Gomez and Gomez (1984).

The rational for analysis of variance is unique in that it compares two different estimates of the population variance to test a hypothesis concerning the population mean. One of these estimates is within-group variance, which is simply the sum of the variances of each of the groups. It is analogous to the used in t tests, extended to the sum of the sample variances of more than two groups. It is called within group variance because it is the collective variance of all observations within each group. The primary feature of the group balanced block design is the grouping of treatments to homogenous blocks based on selected characteristics of the treatments. Whereas the lattice design achieves homogeneity within blocks by grouping experimental units based on some known patterns of heterogeneity in the experimental area, the group balanced block designs achieves the same objective by groping treatments based on some known characteristics of the treatments.

In a group balanced block design, treatments belonging to the same group are always tested in the same block, but those belonging to different groups are never tasted in the same block. Hence, the precision with which the different treatments are compared is not the same for all comparisons. Treatments belonging to the same group are compared with a higher degree of precision than those belonging to different groups. The group balanced block design is commonly used in variety trials where varieties with similar morphological characters are put together in the same group e.g. treatments are grouped based on Growth duration and plant height (Gomez and Gomez 1984).

\subsection{Relative Efficiency and Precision}

Incomplete block design, with its reduced block size, is expected to give a higher degree of precision than a complete block design. Thus, the use of an incomplete block design should generally be preferred so long as the 
resources required for its use (e.g., more replications, inflexible number of treatments, and more complex analysis) can be satisfied (Stringer et al., 2012).

According to Masood et al. (2008) the results of the experiments shown that, there is large difference between error mean squares (EMS) under alpha design and RCB design. The coefficient of variation (CV) of alpha lattice design is comparatively low as compared to RCBD. Low value of CV indicates good index of reliability. The relative efficiency indicates how much more efficient the alpha lattice design is as compared to $\mathrm{RCBD}$, if the value of relative efficiency is greater than one then the alpha lattice results in a smaller error variance and it adjusts genotype means for block effects. In addition to that the relative efficiency is less than one, the alpha lattice design is less efficient than the RCBD. In this case, the trail is analyzed as a RCBD and means are not adjusted for block effects. There is big difference between standard error of difference under RCBD and average standard error of difference under alpha design.

The smaller values of standard error difference for alpha lattice design helps to detect smaller differences for the comparisons of mean. The value of relative efficiency greater than one for both the experiments show that Alpha lattice design was clearly more efficient than RCBD (table1). Relative efficiency indicates that the use of alpha lattice design instead of RCBD increased experimental precision by 24 and 46 percent in wheat and potato respectively.

Table 4. Complete and incomplete designs with their Coefficient of variation, standard error and relative efficiency.

\begin{tabular}{|c|c|c|c|c|c|c|c|}
\hline \multirow[t]{2}{*}{ Exp. } & \multicolumn{2}{|c|}{ Mean square error } & \multicolumn{2}{|c|}{ C.V } & \multicolumn{2}{|c|}{ S.E. (diff) } & \multirow[t]{2}{*}{ R.E } \\
\hline & Alpha & RCBD & Alpha & RCBD & Alpha & RCBD & \\
\hline \multicolumn{8}{|c|}{ Year 2006-07 } \\
\hline Wheat & 95390 & 117841 & 7.70 & 8.54 & 218 & 343 & 1.24 \\
\hline \multicolumn{8}{|c|}{ Year 2007-08 } \\
\hline Potato & 2.072 & 3.016 & 13.6 & 16.39 & 0.831 & 1.418 & 1.46 \\
\hline
\end{tabular}

Source; Raza and Masood (2009)

According to Raza and Masood (2009) who reported that, three datasets were analyzed with Lattice design and randomized complete block design (RCBD). The results of the first dataset showed $26 \%$ precision increased with Lattice design over RCBD. Coefficient of variation of lattice design was $19 \%$ while that of RCBD was $21 \%$, which proves the efficiency of Lattice design. In addition, larger F- value of Lattice design indicates greater variability among the treatments as compared to RCBD. According to the Author, results of the second dataset signify that Lattice design increases the precision of experiment by $17 \%$ and also shows less coefficient of variation than RCBD which implies that lattice design is again more efficient. In third dataset, Lattice design is again more efficient than RCBD in terms of relative efficiency (55\%) and C.V (7\%). The relative efficiencies of three datasets were $26 \%, 17 \%$ and $55 \%$, respectively, and specify that the precision of experiment increased significantly using Lattice design.

Recent developments in several countries showed that considerable improvement in precision can be attained by using alpha lattice design. Generally, the greater the heterogeneity within blocks, the poorer the precision of variety effect estimates. Incomplete block designs are arranged in relatively small blocks that contain fewer varieties than the total number of varieties to be compared (Kempton et al., 1994). Consequently, there is a gain in precision due to use of small blocks. Because of large number of treatments, the homogeneity among experimental units/plots within a large block cannot be maintained. As a result, estimate of experimental error is inflated and results are low in precision (Masood et al., 2008).

The mean square error from each analysis was used to estimate the relative efficiency of a design compared with an RCBD, according to the following equation:

\section{Relative Efficiency $=\underline{\text { Mean Square Error in }(R C B D)} * 100$ \\ Mean Square Error in other Design}

The improved precision with the use of an incomplete block design is achieved with some costs. The major ones are:

$\checkmark \quad$ Inflexible number of treatments or replications or both

$\checkmark$ Unequal degrees of precision in the comparison of treatment means

$\checkmark \quad$ Complex data analysis

In another study conducted to evaluate the relative efficiency of Alpha Lattice Design (ALD) in comparison to 
randomized complete block design. The results indicated that randomized complete block (RCB) design should be replaced by alpha lattice when treatments exceed ten due to the less reliability of homogenous blocks under circumstances. This shows that Alpha Lattice design provide better control on experimental variability among the experimental units under field conditions. Improvement in the precision level in terms of decline in the mean square error, coefficient of variation and standard error of difference were recorded for the ALD.

Table 5. The relative efficiency and coefficient of variation in RCBD and Alpha lattice design.

\begin{tabular}{|l|l|l|l|l|l|l|}
\hline \multirow{2}{*}{ Designs } & \multicolumn{9}{|c|}{ Crop types } \\
\cline { 2 - 7 } & Wheat & Maize & \multicolumn{2}{l|}{ Potato } \\
\cline { 2 - 7 } & CV & RE & CV & RE & CV & RE \\
\hline Alpha Lattice Design & $\mathbf{9 . 2 0}$, & $1.49(49 \%)$ & $\mathbf{1 7 . 8}$ & $1.47(47 \%)$ & $\mathbf{1 4 . 5}$ & $1.34(34 \%)$ \\
\hline RCBD & $\mathbf{1 7 . 3 2}$, & & $\mathbf{2 3 . 7 0}$ & & $\mathbf{1 8 . 5 3})$ & \\
\hline
\end{tabular}

Source: Muhammad et al. (2015).

As indicated from (Table 5), the coefficient of variation (CV) calculated for wheat, maize and potato yield trials were $(9.20,17.8$ and 14.5) for alpha lattice and (17.32, 23.70 and 18.53) for RCB design respectively. The standard error of mean squares calculated for these trials were (292, 3.67 and 2.41) for alpha lattice and (437, 5.40 and 3.23) for RCB design respectively (Muhammad et al., 2015).

In the other study conducted at agricultural experiment and research station in Egypt for two years to evaluate the Efficiency of classical complete and incomplete block designs in yield trial on bread wheat genotypes (Mohamed et al., 2014). In this experiment Fifty-four bread wheat (Triticum aestivum L.) genotypes were included in an alpha lattice design with two replications for seven traits.

Table 6. Estimates of EMS, CV \& RE of Alpha lattice and RCBD

\begin{tabular}{|c|c|c|c|c|c|c|c|c|c|c|c|}
\hline & \multicolumn{5}{|c|}{$2011 / 2012$ season } & \multicolumn{5}{|c|}{$2012 / 2013$ season } & \multirow{3}{*}{$\begin{array}{l}\text { Average } \\
\text { R. E. }\end{array}$} \\
\hline & \multicolumn{2}{|c|}{ Error mean squares } & \multicolumn{2}{|l|}{ CV\% } & \multirow[t]{2}{*}{ R.E } & \multicolumn{2}{|c|}{ Error mean square } & \multicolumn{2}{|l|}{$\mathrm{CV} \%$} & \multirow[t]{2}{*}{ R.E } & \\
\hline & RCBD & Alpha & RCBD & Alpha & & RCBD & Alpha & RCBD & Alpha & & \\
\hline Plant height & 12.05 & 8.67 & 3.50 & 2.97 & 1.39 & 17.72 & 19.44 & 5.30 & 5.56 & 0.91 & 1.15 \\
\hline Spike length & 0.68 & 0.68 & 11.44 & 11.49 & 0.99 & 1.07 & 1.06 & 11.51 & 11.45 & 1.01 & 1.00 \\
\hline $\begin{array}{l}\text { No, of } \\
\text { spikelets/spike }\end{array}$ & 1.76 & 1.57 & 9.33 & 8.82 & 1.12 & 2.75 & 2.46 & 9.33 & 8.82 & 1,12 & 1,12 \\
\hline $\begin{array}{l}\text { No. of } \\
\text { spikes/m2 }\end{array}$ & 18338.80 & 13260.16 & 24.62 & 20.94 & 1.38 & 10035.22 & 6665.63 & 15.41 & 12.56 & 1.51 & 1.44 \\
\hline $\begin{array}{l}\text { no. of grains } \\
\text { /spike }\end{array}$ & 26.92 & 13.23 & 12.59 & 8.82 & 2.04 & 24.72 & 22.12 & 9.33 & 8.82 & 1.12 & 1.58 \\
\hline $\begin{array}{l}\text { Grain } \\
\text { yield/plot }\end{array}$ & 0.17 & 0.16 & 19.67 & 19.85 & 1.04 & 0.64 & 0.50 & 22.20 & 19.71 & 1.27 & 1.13 \\
\hline $\begin{array}{l}\text { Grain yield } \\
\text { ardab /fed. }\end{array}$ & 9.32 & 9.15 & 19.85 & 19.67 & 1.02 & 35.05 & 27.62 & 22.26 & 19.76 & 1.27 & 1.14 \\
\hline
\end{tabular}

Source: Muhammad et al. (2014)

The aim was to assess the efficiency of two experimental designs in minimizing experimental error, coefficient of variation and error mean square for yield variable.

For this purpose, data were analyzed according to alpha lattice design and randomized complete blocks design (RCBD). The results show improvements in the precision level thought decline in the mean square error and coefficient of variation. The relative efficiency (R.E.) of trials shows that alpha lattice design was more efficient than RCBD. the average R.E. results indicates that the use of alpha lattice design instead of RCBD increased experimental accuracy by $15,12,44,58,13$, and $14 \%$ for plant height, number of spikelets/spike, number of spikes/m2, number of grains/spike, grain yield/plot and grain yield (ton/ha), respectively. The rank of genotypes across the two designs and seasons were not constant. Generally, the results showed that the traditional RCBD should be replaced by alpha lattice in the agricultural field trials when the number of treatments to be tested in an experiment increases to more than sixteen, where a homogeneous block is quite difficult to find in field experiments (Muhammad et al., 2014).

\section{SUMMERY}

Experimental designs are known to be used both in the field, green house, lath house and in laboratory according to the type of experiment proposed. Proper design for a given experiment is the most effective way for correct data analysis and interpretation. There are two types of experimental designs mostly known in agriculture i.e. complete block and incomplete block designs. Complete block designs are designs which are used when all treatments are found in each block. The randomized block, Latin square, and other complete block types of experiments are inefficient for large number of treatments, because of their failure to adequately minimize the effect of soil heterogeneity. Generally, the greater the heterogeneity within blocks, the poorer the precision of variety effect estimates.

Incomplete block designs are arranged in relatively small blocks that contain fewer varieties than the total 
number of varieties to be compared. Consequently, there is a gain in precision due to use of small blocks. As far as the layout of the experiment is concerned the incomplete block designs are no more difficult than randomized blocks. Some extra planning is involved in drawing up and randomizing the experimental plan. It is always useful to use incomplete block design when the number of varieties/treatments increases. Because of large number of treatments, the homogeneity among experimental units/plots within a large block cannot be maintained.

Each design has its own advantage and disadvantage and their summery in the form of comparison is stated as the following.

Table 7. Complete block VS incomplete block designs

\begin{tabular}{|l|l|}
\hline $\begin{array}{l}\text { Complete block designs (CRD,RCBD,LS design } \\
\text { and SPD) }\end{array}$ & Incomplete block designs (lattice and augmented) \\
\hline $\begin{array}{l}\text { Require high/ large experimental resources(seed, plot } \\
\text { of land fertilizer and any management costs) }\end{array}$ & Require less/low experimental resources \\
\hline $\begin{array}{l}\text { Inefficient when high number of treatments are } \\
\text { used/can't accommodate } \\
\text { Large number of treatments }\end{array}$ & $\begin{array}{l}\text { Important/efficient for large number of treatments } \\
\text { because of all treatment's don't appear in all blocks } \\
\text { and reduced no of replication }\end{array}$ \\
\hline Calculation/data analysis is not complex & Data analysis is so complex \\
\hline Missed data can be calculated & Missed data couldn't calculated \\
\hline $\begin{array}{l}\text { Less precision and relative efficiency than incomplete } \\
\text { block designs }\end{array}$ & More precise than complete block designs \\
\hline $\begin{array}{l}\text { Treatment comparison is Less affected (if we used } \\
\text { unequal replication in CRD) }\end{array}$ & Treatment comparison is affected by incomplete block \\
\hline Used for both single factor and factorial experiments. & Used only for single factor experiment \\
\hline
\end{tabular}

\section{REFERENCES}

Box, G.E.P., Hunter, J.S., Hunter, W.G. 2005. Statistics for experimenters: Design, innovation, and discovery. 2nd ed. Wiley-Interscience, NY

Casler, M.D. 2013. Finding Hidden Treasure: A 28-Year Case Study for Optimizing Experimental Designs. Communications in Biometry and Crop Science 8 (1), 23-38.

Christensen. R. 2013. Basic experimental designs. Completely randomized designs (CRDs), randomized complete block (RCB) designs, Latin square (LS) designs, balanced incomplete block (BIB) designs, and more. Unbalanced Analysis of Variance, Design, and Regression

Cochran, WG. and Cox, DR. 1957. Experimental designs (Second Edition). John Wiley \& Sons, New York.

Gomez, K.A. and Gomez, A.A. 1984. Statistical Procedures for Agricultural Research.

Parsad Rajender \& Gupta, Vk. 2014. Fundamentals of Design of Experiments. New Delhi.

Hinkelmann, K., Kempthorne, O. 2008. Design and analysis of experiments. I. Introduction to experimental design. 2nd ed. Wiley-Interscience, NY.

Idrees, N. and Khan M.I. 2009. Design improvement using uniformity trials experimental data. Pak. J. Agri. Sci., 46(4): $315-320$

IPGRI. 2001. The design and analysis of evaluation trials of genetic resources collections. A guide for gene bank managers. IPGRI Technical Bulletin No. 4. International Plant Genetic Resources Institute, Rome, Italy.

Katsileros. A, and Koukouvinos. C. 2015. Evaluation of experimental designs in durum wheat trials.

Kempton, R.A., Seraphin, J.C., and Sword, A.M., 1994. Statistical analysis of two dimensional variation in variety yield trials. Journal of Agricultural Science, Cambridge, 122,335-342.

Kelechi. C.A. 2012. Symmetric and Unsymmetrical Balanced Incomplete Block Designs: A Comparative Analysis. International Journal of Statistics and Applications, Nigeria.

Lentner, M. and Bishop, T. 1993. Experimental design and analysis (Second Edition). Valley Book Company, Blacksburg, Virginia

Masood A. A., M. Yaqoob, M. I. Khan and A., Saleem. 2006. Improving Precisions of Agricultural Field Experiments. Journal of Sustainable Development, Vol.3 (1,2), 11-13.

Masood. A.M, Farooq.k, Mujahid.Y and Zubair.M. 2008. Improvement in Precision of Agricultural Field Experiments through Design and analysis. Pakistan journal of life science and social science. Pak. j. life soc. sci. Islamabad-Pakistan.

Muhammad. Ilyas Khan, Asghar S., Ali Shah, Murtaza Khan, Kalim Ullah, Rehmat Ullah, Shahid Iqbal Khatak. 2015. Comparative Efficiency of Alpha Lattice Design and complete Randomized Block Design in Wheat, Maize and Potato field trials. Journal of Resources Development and Management. Vol.11, www.iiste.org.

Nokoe Sagary. 2017. Statistical and Experimental Design Considerations in Alley Farming, Livestock policy analysis. Technical Paper 7: Produced by ILRI.

Raza. I and Masood. M. A. 2009. Efficiency of lattice design in relation to randomized complete block design in 
agricultural field experiments. Pakistan J. Agric. Res. Vol. 22 (3-4), Pakistan.

Stringer J.K., Smith A.B., Cullis B.R. 2012: Spatial analysis of agricultural field experiments. In: K. Hinkelmann (Eds), Design and analysis of experiments, Vol 3, Special designs and applications: 109-136.

Van Es. H.M., Gomes. C.P., Sellmann. M., van Es. C. L. 2009. Spatially-Balanced Complete Block designs for field experiments. Geoderma, 140:346-352. United States.

Yang, R., Z.Y. Terrance, S.B. Stanford and Manjula B. 2004. Efficiency of spatial analyses of field pea variety trials. Crop Sci., 44(1): 49-55. 